\title{
THE BI-DIMENSIONAL SPACE OF KORENBLUM AND COMPOSITION OPERATOR
}

\author{
José A. Guerrero ${ }^{1}$ — Nelson Merentes — José L. SÁnchez

\begin{abstract}
In this paper we present the concept of total $\kappa$-variation in the sense of Hardy-Vitali-Korenblum for a real function defined in the rectangle $I_{a}^{b} \subset \mathbb{R}^{2}$. We show that the space $\kappa B V\left(I_{a}^{b}, \mathbb{R}\right)$ of real functions of two variables with finite total $\kappa$-variation is a Banach space endowed with the norm $\|f\|_{\kappa}=|f(a)|+\kappa T V\left(f, I_{a}^{b}\right)$. Also, we characterize the Nemytskij composition operator $H$ that maps the space of functions of two real variables of bounded $\kappa$-variation $\kappa B V\left(I_{a}^{b}, \mathbb{R}\right)$ into another space of a similar type and is uniformly
\end{abstract} \\ bounded (or Lipschitzian or uniformly continuous).
}

\section{Introduction}

The function of bounded variation was discovered by J o r d a n in 1881 while working out the proof of Dirichlet concerning the convergence of Fourier Series. This concept has been generalized in many ways since then. The desire to extend this theorem to larger classes of functions has provided much of the impetus for the study of generalizations of bounded variation. Following this idea, in 1975, B. Kor e m blu n distorted the measurement of intervals in the domain instead of the range space, as there had been the common practice, to arrive at the concept of $\kappa$-variation.

In 1982 J. Matk ow ski (see [6]) showed that we cannot apply the Banach-Caccioppoli contraction mapping principle in the space $\operatorname{Lip}[a, b]$ to find the solutions to nonlinear equations. More specifically, Matk ow ski showed that the composition operator $H$ generated by a function $h:[a, b] \times \mathbb{R} \longrightarrow \mathbb{R}$ acts and

(C) 2015 Mathematical Institute, Slovak Academy of Sciences.

2010 Mathematics Subject Classification: 47B33, 26B30, 26B40.

Keywords: $\kappa$-function, $\kappa$-variation, composition operator, regularization, Jensen equation, uniformly continuous and bounded operator.

This research has been partly supported by Central Bank of Venezuela.

${ }^{1}$ Partially funded by the Decanato de Investigación-Universidad Nacional Experimental del Táchira-Venezuela - Project 04/004/2010. 
is globally Lipschitzian in the space $\operatorname{Lip}[a, b]$ if and only if

$$
h(t, x)=g_{1}(t) x+g_{2}(t), \quad t \in[a, b], x \in \mathbb{R},
$$

where $g_{1}, g_{2} \in \operatorname{Lip}[a, b]$. The representation (1) is known as Matkowski condition.

There exists a vast literature on the problem treated in this article, mainly in spaces of bounded variation (for instance [3], 7], [10, 11]). In 1984, J. M a tkow ski and J. Miś (see [8]) showed that representation (11) holds in the space of functions of bounded variation $B V[a, b]$ substituting the function $h$ by its left regularization $h^{-}$, that is

$$
h^{-}(t, x)=g_{1}(t) x+g_{2}(t), \quad t \in[a, b], x \in \mathbb{R},
$$

where $g_{1}, g_{2} \in B V[a, b]$. The representation (2) is a weak version of (11) and is called the weak Matkowski condition.

In the present paper we introduce the notion of a function of bounded $\kappa$-variation in the sense of Hardy-Vitali-Korenblum, and we show that the space $\kappa B V\left(I_{a}^{b}, \mathbb{R}\right)$ of the functions of two variables of bounded total variation is a Banach space endowed with the norm $\|f\|_{\kappa}=|f(a)|+\kappa T V\left(f, I_{a}^{b}\right)$. We also show that, if the Nemytskij composition operator $H: \mathbb{R}^{I_{a}^{b}} \longrightarrow \mathbb{R}^{I_{a}^{b}}$ generated by $h$ maps the space $\kappa_{1} B V\left(I_{a}^{b}, \mathbb{R}\right)$ into $\kappa_{2} B V\left(I_{a}^{b}, \mathbb{R}\right)$ and is uniformly bounded, then the one-sided regularization of the generator is affine in the third variable, that is, the composition operator $H$ satisfies the weak Matkowski condition.

\section{Mappings of bounded $\kappa$-variation in $\mathbb{R}^{2}$}

Let: $a=\left(a_{1}, a_{2}\right), b=\left(b_{1}, b_{2}\right) \in \mathbb{R}^{2} ; I_{a}^{b}=\left[a_{1}, b_{1}\right] \times\left[a_{2}, b_{2}\right] \subset \mathbb{R}^{2} ; \xi=\left\{t_{i}\right\}_{i=0}^{m}$ and $\eta=\left\{s_{j}\right\}_{j=0}^{n}$ be partitions of the intervals $\left[a_{1}, b_{1}\right]$ and $\left[a_{2}, b_{2}\right]$, respectively, denoted by $\xi: a_{1}=t_{0}<t_{1}<\cdots<t_{m}=b_{1}$; and $\eta: a_{2}=s_{0}<s_{1}<\cdots<s_{n}=b_{2}$. In the sequel, the symbol $I_{a}^{b}$ denotes the basic rectangle $\left[a_{1}, b_{1}\right] \times\left[a_{2}, b_{2}\right]$ and $\mathbb{R}^{I_{a}^{b}}$ denotes the family of all functions $f: I_{a}^{b} \rightarrow \mathbb{R}$.

Definition 1.1. A $\kappa$-function is a function $\kappa:[0,1] \longrightarrow[0,1]$ with the following properties

(a) $\kappa$ is continuous with $\kappa(0)=0, \kappa(1)=1$,

(b) $\kappa$ is concave and strictly increasing, and

(c) $\lim _{t \longrightarrow 0^{+}} \frac{\kappa(t)}{t}=\infty$.

Now, we give some examples that illustrate Definition 1.1. Important special cases for choices of $\kappa$ are

$$
\kappa_{0}(t)= \begin{cases}t(1-\lg (t)) & \text { if } t \neq 0 \\ 0 & \text { if } \quad t=0\end{cases}
$$


used by B. Korenblum in [4] and

$$
\kappa_{\alpha}(t)=t^{\alpha}, \quad 0<\alpha<1,
$$

in the literature now referred to as the Gevrey case.

The set of all $\kappa$-functions will be denoted by $\mathcal{K}$.

Remark 1.2. Every $\kappa$-function $\kappa$ is subadditive. Then, for all partitions $\xi=\left\{t_{i}\right\}_{i=0}^{m}$ of $[a, b]\left(a=t_{0}<t_{1}<\cdots<t_{m}=b\right)$, one has that

$$
1=\kappa(1)=\kappa\left(\sum_{i=1}^{m} \frac{\left|t_{i}-t_{i-1}\right|}{b-a}\right) \leq \sum_{i=1}^{m} \kappa\left(\frac{\left|t_{i}-t_{i-1}\right|}{b-a}\right) .
$$

For each function $f \in \mathbb{R}^{I_{a}^{b}}$, the following notations are usually used:

$$
\begin{aligned}
\Delta_{10} f\left(t_{i}, s_{j}\right) & =f\left(t_{i}, s_{j}\right)-f\left(t_{i-1}, s_{j}\right), \\
\Delta_{01} f\left(t_{i}, s_{j}\right) & =f\left(t_{i}, s_{j}\right)-f\left(t_{i}, s_{j-1}\right), \\
\Delta_{11} f\left(t_{i}, s_{j}\right) & =f\left(t_{i-1}, s_{j-1}\right)-f\left(t_{i-1}, s_{j}\right)-f\left(t_{i}, s_{j-1}\right)+f\left(t_{i}, s_{j}\right) .
\end{aligned}
$$

\section{Total bounded $\kappa$-variation in the sense of Korenblum in $\mathbb{R}^{2}$}

In this section, we generalize the concept of $\kappa$-variation in $[a, b]$, presented by B. K o r e n blu m in [4, to the two-dimensional Korenblum total $\kappa$-variation in $I_{a}^{b}$, and we prove that the Hardy-Vitali-Korenblum space $\kappa B V\left(I_{a}^{b}, \mathbb{R}\right)$ of the function $f \in \mathbb{R}^{I_{a}^{b}}$ with bounded two-dimensional Korenblum total $\kappa$-variation is a Banach space.

Definition 2.1. The (two-dimensional) total bounded $\kappa$-variation in the sense of Hardy-Vitali-Korenblum is defined as follows: Let $f \in \mathbb{R}^{I_{a}^{b}}$ and $\kappa \in \mathcal{K}$.

(i) Given $x_{2} \in\left[a_{2}, b_{2}\right]$, we defined the Jordan $\kappa$-variation of the function $f\left(\cdot, x_{2}\right)$ in $\left[x_{1}, y_{1}\right] \subset\left[a_{1}, b_{1}\right]$, denoted by $\kappa V_{\left[x_{1}, y_{1}\right]}(f)=\kappa V_{\left[x_{1}, y_{1}\right]}\left(f\left(\cdot, x_{2}\right)\right)$, as

$$
\kappa V_{\left[x_{1}, y_{1}\right]}\left(f\left(\cdot, x_{2}\right)\right):=\sup _{\xi} \frac{\sum_{i=1}^{m}\left|\Delta_{10} f\left(t_{i}, x_{2}\right)\right|}{\sum_{i=1}^{m} \kappa\left(\frac{\left|t_{i}-t_{i-1}\right|}{b_{1}-a_{1}}\right)},
$$

where the supremum is taken over all the partitions $\xi=\left\{t_{i}\right\}_{i=0}^{m}$ of $\left[x_{1}, y_{1}\right]$.

(ii) Given $x_{1} \in\left[a_{1}, b_{1}\right]$, we defined the Jordan $\kappa$-variation of the function $f\left(x_{1}, \cdot\right)$ in $\left[x_{2}, y_{2}\right] \subset\left[a_{2}, b_{2}\right], \kappa V_{\left[x_{2}, y_{2}\right]}(f)=\kappa V_{\left[x_{2}, y_{2}\right]}\left(f\left(x_{1}, \cdot\right)\right)$, as

$$
\kappa V_{\left[x_{2}, y_{2}\right]}\left(f\left(x_{1}, \cdot\right)\right):=\sup _{\eta} \frac{\sum_{j=1}^{n}\left|\Delta_{01} f\left(x_{1}, s_{j}\right)\right|}{\sum_{j=1}^{n} \kappa\left(\frac{\left|s_{j}-s_{j-1}\right|}{b_{2}-a_{2}}\right)},
$$

where the supremum is taken over all the partitions $\eta=\left(s_{j}\right)_{j=0}^{n}$ of $\left[x_{2}, y_{2}\right]$. 
(iii) The two-dimensional Hardy-Vitali-Wiener $\kappa$-variation of the function $f \in \mathbb{R}^{I_{a}^{b}}$, denoted by $\kappa V_{I_{a}^{b}}\left(f, I_{a}^{b}\right)$, is defined as

$$
\kappa V_{I_{a}^{b}}(f):=\sup _{(\xi, \eta)}\left(\frac{\sum_{i=1}^{m} \sum_{j=1}^{n}\left|\Delta_{11} f\left(t_{i}, s_{j}\right)\right|}{\sum_{i=1}^{m} \sum_{j=1}^{n} \kappa\left(\frac{\left|t_{i}-t_{i-1}\right|\left|s_{j}-s_{j-1}\right|}{\left(b_{1}-a_{1}\right)\left(b_{2}-a_{2}\right)}\right)}\right),
$$

where the supremum is taken over all the partitions $(\xi, \eta)$ of $I_{a}^{b}$.

(iv) The total $\kappa$-variation of $f \in \mathbb{R}^{I_{a}^{b}}$ is defined by

$$
\kappa T V\left(f, I_{a}^{b}\right):=\kappa V_{\left[a_{1}, b_{1}\right]}\left(f\left(\cdot, a_{2}\right)\right)+\kappa V_{\left[a_{2}, b_{2}\right]}\left(f\left(a_{1}, \cdot\right)\right)+\kappa V_{I_{a}^{b}}(f) .
$$

(v) A function $f \in \mathbb{R}^{I_{a}^{b}}$ is of the total bounded $\kappa$-variation in $I_{a}^{b}$ if

$$
\kappa T V\left(f, I_{a}^{b}\right)<\infty .
$$

(vi) We will denote the set of all functions $f \in \mathbb{R}^{I_{a}^{b}}$ of the total bounded $\kappa$-variation on $I_{a}^{b}$ by $\kappa B V\left(I_{a}^{b}, \mathbb{R}\right)$, i.e.,

$$
\kappa B V\left(I_{a}^{b}, \mathbb{R}\right)=\left\{f \in \mathbb{R}^{I_{a}^{b}}: \kappa T V\left(f, I_{a}^{b}\right)<\infty\right\} .
$$

Remark 2.2. The set $\kappa B V\left(I_{a}^{b}, \mathbb{R}\right)$ is non-empty since the constant function belongs to it.

In the following result, we establish some relations between these families of functions.

TheOREM 2.3. Let $\kappa, \kappa_{1}, \kappa_{2} \in \mathcal{K}$.

(a) $B V\left(I_{a}^{b}, \mathbb{R}\right)\left[\subset \kappa B V\left(I_{a}^{b}, \mathbb{R}\right)\right.$.

(b) If $\kappa_{1} \leq \kappa_{2}$, then $\kappa_{1} B V\left(I_{a}^{b}, \mathbb{R}\right) \subset \kappa_{2} B V\left(I_{a}^{b}, \mathbb{R}\right)$.

Now, we provide the class $\kappa B V\left(I_{a}^{b}, \mathbb{R}\right)$ of a structure of norm space.

In $\kappa B V\left(I_{a}^{b}, \mathbb{R}\right)$, we define the functional $\|\cdot\|_{\kappa}: \kappa B V\left(I_{a}^{b}, \mathbb{R}\right) \longrightarrow \mathbb{R}$ by

$$
\|f\|_{\kappa}:=|f(a)|+\kappa T V\left(f, I_{a}^{b}\right), \quad a=\left(a_{1}, a_{2}\right) .
$$

TheOREM 2.4. Let $\kappa \in \mathcal{K}$. Then $\left(\kappa B V\left(I_{a}^{b}, \mathbb{R}\right),\|\cdot\|_{\kappa}\right)$ is a normed space. Even more, $\kappa B V\left(I_{a}^{b}, \mathbb{R}\right)$ is a Banach space endowed with the norm $\|\cdot\|_{\kappa}$.

P r o of. Let $\left(f_{\ell}\right)_{\ell \geq 1}$ be a Cauchy sequence in $\left(\kappa B V\left(I_{a}^{b}\right),\|\cdot\|_{\kappa}\right)$. Then for each $\epsilon>0$, there is a positive integer $N$ such that

$$
\left\|f_{\ell}-f_{\ell+1}\right\|_{\kappa}<\epsilon \quad \text { for } \quad \ell \geq N
$$

\footnotetext{
${ }^{1}$ The class of the function $f \in \mathbb{R}^{I_{a}^{b}}$ of bidimensional bounded total variation has been denoted by $B V\left(I_{a}^{b}, \mathbb{R}\right)($ see [2]).
} 


\section{COMPOSITION OPERATORS AND THE SPACE OF KORENBLUM}

Without loss of generality, we assume that $f_{\ell}\left(a_{1}, a_{2}\right)=0$ for all $\ell \geq 1$. Therefore,

Hence,

$$
\kappa T V\left(f_{\ell}-f_{\ell+1}, I_{a}^{b}\right)<\epsilon, \quad \text { for all } \ell \geq N .
$$

$$
\begin{aligned}
& \kappa V_{\left[a_{1}, b_{1}\right]}\left(\left(f_{\ell}-f_{\ell+1}\right)\left(\cdot, a_{2}\right)\right)<\epsilon, \\
& \kappa V_{\left[a_{2}, b_{2}\right]}\left(\left(f_{\ell}-f_{\ell+1}\right)\left(a_{1}, \cdot\right)\right)<\epsilon,
\end{aligned}
$$

and

$$
\kappa V_{I_{a}^{b}}\left(f_{\ell}-f_{\ell+1}\right)<\epsilon, \quad \text { for all } \ell \geq N .
$$

For the partitions $\xi: a_{1}=t_{0}<t=t_{1}<t_{2}=b_{1} ; \eta: a_{2}=s_{0}<s=s_{1}<s_{2}=b_{2}$ of $\left[a_{1}, b_{1}\right]$ and $\left[a_{2}, b_{2}\right]$, respectively, by (44) and the definition of $\kappa$-variation, we get

and

$$
\begin{gathered}
\left|f_{\ell}\left(t, a_{2}\right)-f_{\ell+1}\left(t, a_{2}\right)\right|<2 \epsilon, \\
\left|f_{\ell}\left(a_{1}, s\right)-f_{\ell+1}\left(a_{1}, s\right)\right|<2 \epsilon
\end{gathered}
$$

$$
\begin{array}{r}
\mid\left(f_{\ell}-f_{\ell+1}\right)\left(a_{1}, a_{2}\right)-\left(f_{\ell}-f_{\ell+1}\right)\left(a_{1}, s\right)-\left(f_{\ell}-f_{\ell+1}\right)\left(t, a_{2}\right) \\
+\left(f_{\ell}-f_{\ell+1}\right)(t, s) \mid<4 \epsilon, \quad \text { for all } \quad \ell \geq N .
\end{array}
$$

Therefore, we obtain

$$
\begin{aligned}
\left|f_{\ell}(t, s)-f_{\ell+1}(t, s)\right| \leq & \left|f_{\ell}\left(t, a_{2}\right)-f_{\ell+1}\left(t, a_{2}\right)\right|+\left|f_{\ell}\left(a_{1}, s\right)-f_{\ell+1}\left(a_{1}, s\right)\right| \\
& +\mid\left(f_{\ell}-f_{\ell+1}\right)\left(a_{1}, a_{2}\right)-\left(f_{\ell}-f_{\ell+1}\right)\left(a_{1}, s\right) \\
& -\left(f_{\ell}-f_{\ell+1}\right)\left(t, a_{2}\right)+\left(f_{\ell}-f_{\ell+1}\right)(t, s) \mid \\
< & 8 \epsilon . \quad \text { for all }(t, s) \in I_{a}^{b},
\end{aligned}
$$

Making use of (5), the sequence $\left(f_{\ell}(t, s)\right)_{\ell \geq 1},(t, s) \in I_{a}^{b}$ is Cauchy in $\mathbb{R}$, so, by completeness of $\mathbb{R}$, there exists a function $f: I_{a}^{b} \longrightarrow \mathbb{R}$ such that

$$
f(t, s)=\lim _{\ell \longrightarrow \infty} f_{\ell}(t, s), \quad \text { for all } \quad(t, s) \in I_{a}^{b} .
$$

Since $\left(f_{\ell}\right)_{\ell \geq 1}$ is a Cauchy sequence in $\left(\kappa B V\left(I_{a}^{b}\right),\|\cdot\|_{\kappa}\right)$ and by (6) , we get

$$
\begin{aligned}
\kappa T V\left(f_{\ell}-f\right)= & \sup _{\xi} \lim _{\ell^{\prime} \longrightarrow \infty} \frac{\sum_{i=1}^{m} \Delta_{10} f_{\ell}\left(t_{i}, a_{2}\right)-\Delta_{10} f_{\ell^{\prime}}\left(t_{i}, a_{2}\right) \mid}{\sum_{i=1}^{m} \kappa\left(\frac{\left|t_{i}-t_{i-1}\right|}{b_{1}-a_{1}}\right)} \\
& +\sup _{\eta} \lim _{\ell^{\prime} \longrightarrow \infty} \frac{\sum_{j=1}^{n}\left|\Delta_{01} f_{\ell}\left(a_{1}, s_{j}\right)-\Delta_{01} f_{\ell^{\prime}}\left(a_{1}, s_{j}\right)\right|}{\sum_{j=1}^{n} \kappa\left(\frac{\left|s_{j}-s_{j-1}\right|}{b_{2}-a_{2}}\right)} \\
& +\sup _{(\xi, \eta)} \lim _{\ell^{\prime} \longrightarrow \infty} \frac{\sum_{i=1}^{m} \sum_{j=1}^{n}\left|\Delta_{11} f_{\ell}\left(t_{i}, s_{j}\right)-\Delta_{11} f_{\ell^{\prime}}\left(t_{i}, s_{j}\right)\right|}{\sum_{i=1}^{m} \sum_{j=1}^{n} \kappa\left(\frac{\left|t_{i}-t_{i-1}\right|\left|s_{j}-s_{j-1}\right|}{\left(b_{1}-a_{1}\right)\left(b_{2}-a_{2}\right)}\right)} \\
\leq & 3 \epsilon, \quad \text { for all } \quad \ell \geq N .
\end{aligned}
$$


By (77), we get that $f \in \kappa B V\left(I_{a}^{b}\right)$ (note that: $f-f_{\ell}, f_{\ell} \in \kappa B V\left(I_{a}^{b}\right)$ and $\left.f=\left(f-f_{\ell}\right)+f_{\ell}\right)$ and $\left\|f_{\ell}-f\right\|_{\kappa}<\epsilon$.

\section{The regularization functions}

In this section, we present the concept of regularizations of real functions of two variables.

Definition 3.1. Let $f \in \mathbb{R}^{I_{a}^{b}}$. We define the left-left regularization $f^{-} \in \mathbb{R}^{I_{a}^{b}}$ of $f$ by

$$
f^{-}\left(x_{1}, x_{2}\right):= \begin{cases}\lim _{y_{1} \rightarrow x_{1}^{-}} f\left(y_{1}, y_{2}\right) & \text { for }\left(x_{1}, x_{2}\right) \in\left(a_{1}, b_{1}\right] \times\left(a_{2}, b_{2}\right], \\ y_{2} \rightarrow x_{2}^{-} & \\ \lim _{y_{1} \rightarrow x_{1}^{-}} f\left(y_{1}, y_{2}\right) & \text { for } x_{1} \in\left(a_{1}, b_{1}\right] \text { and } x_{2}=a_{2}, \\ y_{2} \rightarrow a_{2}^{+} & \\ \lim _{y_{1} \rightarrow a_{1}^{+}} f\left(y_{1}, y_{2}\right) & \text { for } x_{1}=a_{1} \text { and } x_{2} \in\left(a_{2}, b_{2}\right], \\ y_{2} \rightarrow x_{2}^{-} & \\ \lim _{y_{1} \rightarrow a_{1}^{+}} f\left(y_{1}, y_{2}\right) & \text { for } x_{1}=a_{1} \text { and } x_{2}=a_{2} . \\ y_{2} \rightarrow a_{2}^{+} & \end{cases}
$$

Definition 3.2. A function $f \in \mathbb{R}^{I_{a}^{b}}$ is said to be left-left continuous if

$$
\lim _{\substack{x \rightarrow t^{-} \\ y \rightarrow s^{-}}} f(x, y)=f(t, s), \quad \text { for all }(t, s) \in\left(a_{1}, b_{1}\right] \times\left(a_{2}, b_{2}\right] .
$$

We denote the subspace of $\kappa B V\left(I_{a}^{b}, \mathbb{R}\right)$ of those functions $f$ which are left-left continuous on $\left(a_{1}, b_{1}\right] \times\left(a_{2}, b_{2}\right]$ by $\kappa B V^{-}\left(I_{a}^{b}, \mathbb{R}\right)$.

LEMmA 3.3. If $f \in \kappa B V\left(I_{a}^{b}, \mathbb{R}\right)$, then $f^{-} \in \kappa B V^{-}\left(I_{a}^{b}, \mathbb{R}\right)$.

P r o of. From the first line of the definition of the left-left regularization, it follows directly that $f^{-}$is left-left continuous on $\left(a_{1}, b_{1}\right] \times\left(a_{2}, b_{2}\right]$. We prove that $\kappa T V\left(f^{-}, I_{a}^{b}\right)<\infty$. In fact, for every $\varepsilon>0$, let $a_{1}=t_{0}<t_{1}<\cdots<t_{m}=b_{1}$ and $a_{2}=s_{0}<s_{1}<\cdots<s_{n}=b_{2}$ be partition of $\left[a_{1}, b_{1}\right]$ and $\left[a_{2}, b_{2}\right]$, respectively. From the definition of $f^{-}$, we obtain that

$$
\begin{aligned}
\kappa V_{\left[a_{1}, b_{1}\right]}\left(f^{-}\left(\cdot, a_{2}\right)\right) & \leq \kappa V_{\left[a_{1}, b_{1}\right]}\left(f\left(\cdot, a_{2}\right)\right)+\epsilon, \\
\kappa V_{\left[a_{2}, b_{2}\right]}\left(f^{-}\left(a_{1}, \cdot\right)\right) & \leq \kappa V_{\left[a_{2}, b_{2}\right]}\left(f\left(a_{1}, \cdot\right)\right)+\epsilon, \\
\kappa V_{I_{a}^{b}}\left(f^{-}\right) & \leq \kappa V_{I_{a}^{b}}(f)+\epsilon .
\end{aligned}
$$

Therefore, $\kappa T V\left(f^{-}, I_{a}^{b}\right)<\infty$. 


\section{COMPOSITION OPERATORS AND THE SPACE OF KORENBLUM}

\section{An auxiliary result and its consequences}

Following the idea given by J. Matkowski in 9, we prove Theorem 4.1

TheOREM 4.1. Let $\kappa_{1}, \kappa_{2} \in \mathcal{K}$ and $h: I_{a}^{b} \times \mathbb{R} \rightarrow \mathbb{R}$ be a continuous function with respect to the third variable. If the composition operator $H: \mathbb{R}^{I_{a}^{b}} \longrightarrow \mathbb{R}^{I_{a}^{b}}$ generated by $h$ maps the space $\kappa_{1} B V\left(I_{a}^{b}, \mathbb{R}\right)$ into $\kappa_{2} B V\left(I_{a}^{b}, \mathbb{R}\right)$ and satisfies the inequality

$$
\left\|H\left(f_{1}\right)-H\left(f_{2}\right)\right\|_{\kappa_{2}} \leq \gamma\left(\left\|f_{1}-f_{2}\right\|_{\kappa_{1}}\right), \quad f_{1}, f_{2} \in \kappa_{1} B V\left(I_{a}^{b}, \mathbb{R}\right),
$$

for some function $\gamma:[0, \infty) \longrightarrow[0, \infty)$, then there are functions

such that

$$
a_{0}, a_{1} \in \kappa_{2} B V^{-}\left(I_{a}^{b}, \mathbb{R}\right),
$$

$$
h^{-}(z, y)=a_{0}(z)+a_{1}(z) y, \quad z=(t, s) \in I_{a}^{b}, y \in \mathbb{R} .
$$

P r o o f. For each $y \in \mathbb{R}$, the constant function $f(z)=y\left(z=(t, s) \in I_{a}^{b}\right)$ belongs to $\kappa_{1} B V\left(I_{a}^{b}, \mathbb{R}\right)$. Since $H$ maps $\kappa_{1} B V\left(I_{a}^{b}, \mathbb{R}\right)$ into $\kappa_{2} B V\left(I_{a}^{b}, \mathbb{R}\right)$, we have that the function $(H f)(z)=h(z, f(z))=h(z, y) \in \kappa_{2} B V\left(I_{a}^{b}, \mathbb{R}\right)$. By Lemma 3.3, the function regularization left-left $h^{-}(\cdot, y) \in \kappa_{2} B V^{-}\left(I_{a}^{b}, \mathbb{R}\right)$ for all $y \in \mathbb{R}$.

For $s^{\prime}, t^{\prime} \in \mathbb{R}, s^{\prime}<t^{\prime}$, let us define the function $\eta_{s^{\prime}, t^{\prime}}: \mathbb{R} \longrightarrow[0,1]$ by

$$
\eta_{s^{\prime}, t^{\prime}}(t):= \begin{cases}0 & \text { if } \quad t \leq s^{\prime}, \\ \frac{t-s^{\prime}}{t^{\prime}-s^{\prime}} & \text { if } \quad s^{\prime} \leq t \leq t^{\prime}, \\ 1 & \text { if } t^{\prime} \leq t .\end{cases}
$$

Let $\mathcal{H}=H f_{1}-H f_{2}$. Fix $r_{1} \in\left(a_{1}, b_{1}\right], r_{2} \in\left(a_{2}, b_{2}\right], m \in \mathbb{N}$, and take an arbitrary finite sequence

$$
\begin{aligned}
& a_{1}<\alpha_{1}<\beta_{1}<\alpha_{2}<\beta_{2}<\cdots<\alpha_{m}<\beta_{m}<r_{1}, \\
& a_{2}<\bar{\alpha}_{1}<\bar{\beta}_{1}<\bar{\alpha}_{2}<\bar{\beta}_{2}<\cdots<\bar{\alpha}_{m}<\bar{\beta}_{m}<r_{2} .
\end{aligned}
$$

For arbitrary numbers $y_{1}, y_{2} \in \mathbb{R}, y_{1} \neq y_{2}$, the functions $f_{1}, f_{2}: I_{a}^{b} \longrightarrow \mathbb{R}$ defined by

$$
f_{\ell}(t, s):=\frac{1}{2}\left[\eta_{\alpha_{i}, \beta_{i}}(t)+\eta_{\bar{\alpha}_{i}, \bar{\beta}_{i}}(s)\right] y_{1}+(2-\ell) y_{2}, \quad(t, s) \in I_{a}^{b}, \quad \ell=1,2,
$$

belong to $\kappa_{1} B V\left(I_{a}^{b}, \mathbb{R}\right)$. From (9), we have

$$
f_{1}-f_{2} \equiv y_{2},
$$

therefore,

Since

$$
\left\|f_{1}-f_{2}\right\|_{\kappa_{1}}=\left|y_{2}\right| \text {. }
$$

$$
\|\mathcal{H}\|_{\kappa_{2}} \geq \frac{\sum_{i=1}^{m}\left|\mathcal{H}\left(\beta_{i}, \bar{\beta}_{i}\right)-\mathcal{H}\left(\alpha_{i}, \bar{\alpha}_{i}\right)\right|}{C_{m}}
$$




\section{JOSÉ A. GUERRERO — NELSON MERENTES — JOSÉ L. SÁNCHEZ}

where

$$
C_{m}=\max \left\{\begin{array}{c}
\sum_{i=1}^{2 m+1} \kappa_{1}\left(\frac{t_{i}-t_{i-1}}{b_{1}-a_{1}}\right), \\
\sum_{j=1}^{2 m+1} \kappa_{1}\left(\frac{s_{j}-s_{j-1}}{b_{2}-a_{2}}\right), \\
\sum_{i=1}^{2 m+1} \sum_{j=1}^{2 m+1} \kappa_{1}\left(\frac{t_{i}-t_{i-1}}{b_{1}-a_{1}} \frac{s_{j}-s_{j-1}}{b_{2}-a_{2}}\right)
\end{array}\right\}
$$

and

$$
\begin{aligned}
& t_{0}=a_{1}, \quad t_{2 k-1}=\alpha_{k}, \quad t_{2 k}=\beta_{k}, \quad k=1, \ldots, m, \quad t_{2 m+1}=b_{1}, \\
& s_{0}=a_{2}, \quad s_{2 k-1}=\bar{\alpha}_{k}, \quad s_{2 k}=\bar{\beta}_{k}, \quad k=1, \ldots, m, \quad s_{2 m+1}=b_{2} .
\end{aligned}
$$

Therefore,

$$
\sum_{i=1}^{m}\left|\mathcal{H}\left(\beta_{i}, \bar{\beta}_{i}\right)-\mathcal{H}\left(\alpha_{i}, \bar{\alpha}_{i}\right)\right| \leq C_{m} \gamma\left(\left|y_{2}\right|\right)
$$

As

$f_{1}\left(\alpha_{i}, \bar{\alpha}_{i}\right)=y_{2}, \quad f_{2}\left(\alpha_{i}, \bar{\alpha}_{i}\right)=0, \quad f_{1}\left(\beta_{i}, \bar{\beta}_{i}\right)=y_{1}+y_{2} \quad$ and $\quad f_{2}\left(\beta_{i}, \bar{\beta}_{i}\right)=y_{1}$,

the last inequality can be rewritten in the form

$$
\begin{array}{r}
\sum_{i=1}^{m}\left|h\left(\beta_{i}, \bar{\beta}_{i}, y_{1}+y_{2}\right)-h\left(\beta_{i}, \bar{\beta}_{i}, y_{1}\right)-h\left(\alpha_{i}, \bar{\alpha}_{i}, y_{2}\right)+h\left(\alpha_{i}, \bar{\alpha}_{i}, 0\right)\right| \\
\leq C_{m} \gamma\left(\left|y_{2}\right|\right) .
\end{array}
$$

So, if $\left(\alpha_{1}, \bar{\alpha}_{1}\right) \longrightarrow\left(r_{1}^{-}, r_{2}^{-}\right)$, then

$$
C_{m} \longrightarrow C=\max \left\{\begin{array}{c}
\kappa_{1}\left(\frac{b_{1}-r_{1}}{b_{1}-a_{1}}\right)+\kappa_{1}\left(\frac{r_{1}-a_{1}}{b_{1}-a_{1}}\right), \\
\kappa_{1}\left(\frac{b_{2}-r_{2}}{b_{2}-a_{2}}\right)+\kappa_{1}\left(\frac{r_{2}-a_{2}}{b_{2}-a_{2}}\right), \\
\kappa_{1}\left(\frac{b_{1}-r_{1}}{b_{1}-a_{1}} \frac{b_{2}-r_{2}}{b_{2}-a_{2}}\right)+\kappa_{1}\left(\frac{b_{1}-r_{1}}{b_{1}-a_{1}} \frac{b_{2}-r_{2}}{b_{2}-a_{2}}\right)+ \\
\kappa_{1}\left(\frac{r_{1}-a_{1}}{b_{1}-a_{1}} \frac{r_{2}-a_{2}}{b_{2}-a_{2}}\right)+\kappa_{1}\left(\frac{r_{1}-a_{1}}{b_{1}-a_{1}} \frac{r_{2}-a_{2}}{b_{2}-a_{2}}\right)
\end{array}\right\}
$$

and

$$
\begin{array}{r}
\sum_{i=1}^{m}\left|h^{-}\left(r_{1}, r_{2}, y_{1}+y_{2}\right)+h^{-}\left(r_{1}, r_{2}, y_{1}\right)-h^{-}\left(r_{1}, r_{2}, y_{2}\right)+h^{-}\left(r_{1}, r_{2}, 0\right)\right| \\
\leq C \gamma\left(\left|y_{2}\right|\right),
\end{array}
$$

whence

$$
\begin{array}{r}
\left|h^{-}\left(r_{1}, r_{2}, y_{1}+y_{2}\right)+h^{-}\left(r_{1}, r_{2}, y_{1}\right)-h^{-}\left(r_{1}, r_{2}, y_{2}\right)+h^{-}\left(r_{1}, r_{2}, 0\right)\right| \\
\leq \frac{C \gamma\left(\left|y_{2}\right|\right)}{m}
\end{array}
$$




\section{COMPOSITION OPERATORS AND THE SPACE OF KORENBLUM}

Since $m \in \mathbb{N}$ is arbitrary, we have

$$
\left|h^{-}\left(r_{1}, r_{2}, y_{1}+y_{2}\right)+h^{-}\left(r_{1}, r_{2}, y_{1}\right)-h^{-}\left(r_{1}, r_{2}, y_{2}\right)+h^{-}\left(r_{1}, r_{2}, 0\right)\right|=0 \text {. }
$$

Therefore,

$$
h^{-}\left(r_{1}, r_{2}, y_{1}+y_{2}\right)+h^{-}\left(r_{1}, r_{2}, y_{1}\right)-h^{-}\left(r_{1}, r_{2}, y_{2}\right)+h^{-}\left(r_{1}, r_{2}, 0\right)=0
$$

for all $r_{1} \in\left(a_{1}, b_{1}\right], r_{2} \in\left(a_{2}, b_{2}\right]$ and $y_{1}, y_{2} \in \mathbb{R}, y_{1} \neq y_{2}$.

For $r_{1} \in\left(a_{1}, b_{1}\right]$ and $r_{2}=a_{2}$, let

and

$$
a_{1}<\alpha_{1}<\beta_{1}<\alpha_{2}<\beta_{2}<\cdots<\alpha_{m}<\beta_{m}<r_{1}
$$

$$
a_{2}<\bar{\alpha}_{1}<\bar{\beta}_{1}<\bar{\alpha}_{2}<\bar{\beta}_{2}<\cdots<\bar{\alpha}_{m}<\bar{\beta}_{m}<b_{2},
$$

the above arguments provide the estimate (10). Taking the limit when

$$
\left(\alpha_{1}, \bar{\beta}_{m}\right) \longrightarrow\left(r_{1}^{-}, a_{2}^{+}\right),
$$

we arrive at equality (11).

The cases when $r_{1}=a_{1}$ and $r_{2} \in\left(a_{2}, b_{2}\right]$ or $r_{1}=a_{1}$ and $r_{2}=a_{2}$ are treated in a similar way. In consequence, equality (11) holds for all $(t, s) \in I_{a}^{b}, y_{1}, y_{2} \in \mathbb{R}$, $y_{1} \neq y_{2}$.

To complete the proof of the theorem, we apply a standard argument (adapted) used in M. K u c z m a [5, pp. 314-315]. For all $z \in I_{a}^{b}$, we define the function $g_{z}: \mathbb{R} \rightarrow \mathbb{R}$ by

$$
g_{z}(y):=h^{-}(z, y)-h^{-}(z, 0), \quad y \in \mathbb{R} .
$$

For all $y_{1}, y_{2} \in \mathbb{R}$, from (11) and (12), we have

$$
\begin{aligned}
g_{z}\left(y_{1}+y_{2}\right) & =h^{-}\left(z, y_{1}+y_{2}\right)-h^{-}(z, 0) \\
& =h^{-}\left(z, y_{1}\right)+h^{-}\left(z, y_{2}\right)-2 h^{-}(z, 0) \\
& =g_{z}\left(y_{1}\right)+g_{z}\left(y_{2}\right) .
\end{aligned}
$$

This shows that $g_{z}$ is an affine Jensen function in $\mathbb{R}$ and $g_{z}(0)=0$.

Let us define the function $f(y)=g_{z}(y), y \in \mathbb{R}$. Since $g_{z}$ is an affine Jensen function, for all $y_{1}, y_{2} \in \mathbb{R}$, we get

$$
f\left(y_{1}+y_{2}\right)=f\left(y_{1}\right)+f\left(y_{2}\right) .
$$

In other words, $f$ is an additive function. Then, the continuity of $h^{-}(z, \cdot)$ implies the continuity of $f$. Consequently, since $f$ is additive, $f$ has to be linear; i.e., there is a function $a_{1}: I_{a}^{b} \longrightarrow \mathbb{R}$ such that

$$
f(y)=g_{z}(y)=a_{1}(z) y
$$

for all $z \in I_{a}^{b}$ and all $y \in \mathbb{R}$. Taking $a_{0}(z)=h^{-}(z, 0)$, we have

and so,

$$
h^{-}(z, y)-h^{-}(z, 0)=g_{z}(y)=f(y)=a_{1}(z) y,
$$

$$
h^{-}(z, y)=a_{0}(z)+a_{1}(z) y, \quad z \in I_{a}^{b}, y \in \mathbb{R} .
$$


Since $a_{0}(\cdot)=h^{-}(\cdot, 0)$ and $a_{1}(\cdot)=h^{-}(\cdot, 1)-h^{-}(\cdot, 0)$, by Lemma 3.3, we obtain $a_{0}, a_{1} \in \kappa_{2} B V^{-}\left(I_{a}^{b}, \mathbb{R}\right)$.

As a consequence of Theorem 4.1, we obtain the following result.

ThEOREM 4.2. Let $\kappa_{1}, \kappa_{2} \in \mathcal{K}$ and $h: I_{a}^{b} \times \mathbb{R} \rightarrow \mathbb{R}$ be a continuous function with respect to the third variable. If the composition operator $H: \mathbb{R}^{I_{a}^{b}} \longrightarrow \mathbb{R}^{I_{a}^{b}}$ generated by $h$ maps the space $\kappa_{1} B V\left(I_{a}^{b}, \mathbb{R}\right)$ into $\kappa_{2} B V\left(I_{a}^{b}, \mathbb{R}\right)$ and

(i) $H$ is Lipschitzian, or

(ii) $H$ is uniformly continuous,

then there are functions $a_{0}, a_{1} \in \kappa_{2} B V^{-}\left(I_{a}^{b}, \mathbb{R}\right)$, such that

$$
h^{-}(z, y)=a_{0}(z)+a_{1}(z) y, \quad z=(t, s) \in I_{a}^{b}, \quad y \in \mathbb{R} .
$$

P r o o f. (i) Since $H$ is a Lipschitzian operator, there is a constant $\mu>0$ such that

$$
\left\|H\left(f_{1}\right)-H\left(f_{2}\right)\right\|_{\kappa_{2}} \leq \mu\left\|f_{1}-f_{2}\right\|_{\kappa_{1}}
$$

for all $f_{1}, f_{2} \in \kappa_{1} B V\left(I_{a}^{b}, \mathbb{R}\right)$.

(ii) On the other hand, taking the uniform continuity of $H$ into account, the modulus of continuity of $H, \gamma:[0, \infty) \longrightarrow[0, \infty)$, i.e.,

$$
\gamma(\tau)=\sup \left\{\left\|H\left(f_{1}\right)-H\left(f_{2}\right)\right\|_{\kappa_{2}}:\left\|f_{1}-f_{2}\right\|_{\kappa_{1}} \leq \tau, f_{1}, f_{2} \in \kappa_{1} B V\left(I_{a}^{b}, \mathbb{R}\right)\right\}
$$

for $\tau \geq 0$, is well defined. Also, from the definition of the $\|\cdot\|_{\kappa}$, we obtain

$$
\left\|H\left(f_{1}\right)-H\left(f_{2}\right)\right\|_{\kappa_{2}} \leq \gamma\left(\left\|f_{1}-f_{2}\right\|_{\kappa_{1}}\right), f_{1}, f_{2} \in \kappa_{1} B V\left(I_{a}^{b}, \mathbb{R}\right) .
$$

Hence, by Theorem 4.1, we get the required result.

\section{Uniformly bounded composition operator}

Definition 5.1 ([9, Def. 1]). Let $X$ and $Y$ be two metric (or normed) spaces. We say that a mapping $H: X \longrightarrow Y$ is uniformly bounded if, for any $t>0$, there is a real number $\gamma(t)$ such that, for any nonempty set $B \subset X$, we have

$$
\operatorname{diam}(B) \leq t \Longrightarrow \operatorname{diam} H(B) \leq \gamma(t) .
$$

Remark 5.2. Obviously, every uniformly continuous operator or Lipschitzian operator is uniformly bounded. Note that, under the assumptions of this definition, every bounded operator is uniformly bounded. 


\section{COMPOSITION OPERATORS AND THE SPACE OF KORENBLUM}

Our main result reads as follows

TheOREM 5.3. Let $\kappa_{1}, \kappa_{2} \in \mathcal{K}$ and $h: I_{a}^{b} \times \mathbb{R} \rightarrow \mathbb{R}$ be a continuous with respect to the third variable. If the composition operator $H: \mathbb{R}^{I_{a}^{b}} \longrightarrow \mathbb{R}^{I_{a}^{b}}$ generated by $h$ maps the space $\kappa_{1} B V\left(I_{a}^{b}, \mathbb{R}\right)$ into $\kappa_{2} B V\left(I_{a}^{b}, \mathbb{R}\right)$ and is uniformly bounded, then there are functions $a_{0}, a_{1} \in \kappa_{2} B V^{-}\left(I_{a}^{b}, \mathbb{R}\right)$, such that

$$
h^{-}(z, y)=a_{0}(z)+a_{1}(z) y, \quad z=(t, s) \in I_{a}^{b}, y \in \mathbb{R} .
$$

P r o of. Take any $\tau \geq 0$ and arbitrary $f_{1}, f_{2} \in \kappa_{1} B V\left(I_{a}^{b}, \mathbb{R}\right)$ such that

$$
\left\|f_{1}-f_{2}\right\|_{\kappa_{1}} \leq \tau \text {. }
$$

Since $\operatorname{diam}\left\{f_{1}, f_{2}\right\} \leq \tau$, by the uniform boundedness of $H$, we have

that is

$$
\operatorname{diam} H\left(\left\{f_{1}, f_{2}\right\}\right) \leq \gamma(\tau),
$$

$$
\left\|H\left(f_{1}\right)-H\left(f_{2}\right)\right\|_{\kappa_{2}}=\operatorname{diam} H\left(\left\{f_{1}, f_{2}\right\}\right) \leq \gamma\left(\left\|f_{1}-f_{2}\right\|_{\kappa_{1}}\right),
$$

and the result follows from Theorem 4.1

Remark 5.4. If the function $\gamma:[0, \infty) \longrightarrow[0, \infty)$ in Definition 5.1 is right continuous at 0 and $\gamma(0)=0$ (or if only $\gamma\left(0^{+}\right)=0$ ) then, clearly, the uniform boundedness of the involved operator reduces to its uniform continuity.

Consider the following:

Definition 5.5 ([9, Def. 2]). Let $X$ and $Y$ be two metric (or normed) spaces. We say that a mapping $H: X \longrightarrow Y$ is equidistantly uniformly bounded if, for any $t>0$, there is a nonnegative real number $\gamma(t)$ such that for all $u, v \in B \subset X$,

$$
\operatorname{diam}\{u, v\}=t \Longrightarrow \operatorname{diam}\{H(u), H(v)\} \leq \gamma(t)
$$

Of course, the equidistant uniform boundedness is a weaker condition than the uniform boundedness. The following result follows from Theorem 4.1

TheOREM 5.6. Let $\kappa_{1}, \kappa_{2} \in \mathcal{K}$ and $h: I_{a}^{b} \times \mathbb{R} \rightarrow \mathbb{R}$ be a continuous function with respect to the third variable. If the composition operator $H: \mathbb{R}^{I_{a}^{b}} \longrightarrow \mathbb{R}^{I_{a}^{b}}$ generated by $h$ maps the space $\kappa_{1} B V\left(I_{a}^{b}, \mathbb{R}\right)$ into $\kappa_{2} B V\left(I_{a}^{b}, \mathbb{R}\right)$ and is equidistantly uniformly bounded, then there are functions $a_{0}, a_{1} \in \kappa_{2} B V^{-}\left(I_{a}^{b}, \mathbb{R}\right)$, such that

$$
h^{-}(z, y)=a_{0}(z)+a_{1}(z) y, \quad z=(t, s) \in I_{a}^{b}, y \in \mathbb{R} .
$$

Acknowledgements. The authors express his gratitude to the referee for his valuable comments and suggestions and also want to give thanks to the library staff of B.C.V. for compiling the references. 


\section{JOSÉ A. GUERRERO — NELSON MERENTES — JOSÉ L. SÁNCHEZ}

\section{REFERENCES}

[1] APPELL, J.-ZABREJKO, P. P.: Nonlinear superposition operators. Cambridge University Press, Cambridge, 1990.

[2] CHISTYAKOV, V. V.: Superposition operators in the algebra of functions of two variables with finite total variation, Monatsh. Math. 137 (2002), 99-114.

[3] EREÚ, T.-MERENTES, N.-SÁNCHEZ, J. L.-WRÓBEL, M.: Uniformly bounded setvalued composition operators in the spaces of functions of bounded variation in the sense of Schramm, Jan Długosz University in Czȩstochowa, Scientific Issues, Mathematics XVII (2012), 37-47.

[4] KORENBLUM, B.: An extension of the Nevanlinna theory, Acta Math. 135 (1975), $187-219$.

[5] KUCZMA, M.: An Introduction to the Theory of Functional Equations and Inequalities. Cauchy's Equation and Jensen's Inequality. PWN, Warszawa, 1985.

[6] MATKOWSKI, J.: Functional equations and Nemytskij operators, Funck. Ekvacioj Ser. Int. 25 (1982), 127-132.

[7] MATKOWSKI, J.: Form of Lipschitz operators of substitution in Banach spaces of differentiable functions, Zeszyty Nauk. Politech. Łódź Mat. 17 (1984), 5-10.

[8] MATKOWSKI, J. -MIŚ, J.: On a characterization of Lipschitzian operators of substitution in the space $B V[a, b]$, Math. Nachr. 117 (1984), 155-159.

[9] MATKOWSKI, J.: Uniformly bounded composition operators between general Lipschitz function normed spaces, Topol. Methods Nonlinear Anal. 38(2) (2011), 395-406.

[10] MATKOWSKA, A.: On the characterization of Lipschitzian operator of substitution in the class Hölder functions, Zeszyty Nauk. Politech. Łódź Mat. 17 (1984), 81-85.

[11] MERENTES, N.: On a characterization of Lipschitzian operators of substitution in the space of bounded Riesz $\varphi$-variation, Annales Univ. Sci. Budapest 34 (1991), 139-144.

Received June 8, 2013
José A. Guerrero

Dpto. de Matemáticas y Física

Universidad Nacional Experimental del Táchira

San Cristóbal

VENEZUELA

E-mail: jaguerrero4@gmail.com jguerre@unet.edu.ve

Nelson Merentes

José L. Sánchez

Escuela de Matemáticas

Universidad Central de Venezuela

Caracas

VENEZUELA

E-mail:nmer@ciens.ucv.ve casanay085@hotmail.com 